\title{
Effect of Carotid Artery Stenting and Extracranial-Intracranial By-Pass on Cognitive Function: Preliminary Results
}

\author{
Li Liu', Yuying Zhou ${ }^{2}$ \\ ${ }^{1}$ Department of Radiology, Tianjin Huan Hu Hospital, Tianjin, China \\ ${ }^{2}$ Department of Neurology, Tianjin Huan Hu Hospital, Tianjin, China \\ Email: zhouyuyinghh@163.com
}

Received 1 May 2015; accepted 1 June 2015; published 5 June 2015

Copyright (C) 2015 by authors and Scientific Research Publishing Inc.

This work is licensed under the Creative Commons Attribution International License (CC BY).

http://creativecommons.org/licenses/by/4.0/

\begin{abstract}
Background: To compare the neuropsychological consequences after carotid artery stenting (CAS) and extracranial-intracranial by-pass (EC-IC by-pass). Methods: A total of 43 patients referred to CAS, 32 patients referred to EC-IC by-pass and 43 control subjects were enrolled in the study. Neuropsychologic testing was performed before and three months after procedure. A paired Student $t$ test was used to compare neuropsychologic test scores at baseline and three months after procedure in each group. Cognitive changes in a three-month follow-up were not normally distributed and compared among/between groups with Kruskal-Wallis test. Results: Three months after the treatment both the CAS and EC-IC by-pass groups showed improved cognitive performance compared to baseline, whereas the same improvement wasn't seen in the control group. The scores from Activities of Daily Living also improved in all three groups in a three-month follow-up, and EC-IC group presented a more distinct increase in daily life abilities comparing to the other two groups. Conclusions: CAS and EC-IC by-pass in patients with a carotid or intracranial stenosis may result in cognitive improvement three months after surgery.
\end{abstract}

\section{Keywords}

Carotid Artery Stenting, Extracranial-Intracranial By-Pass, Cognition

\section{Introduction}

Atherosclerosis of the extracranial and intracranial artery is an important mechanism underlying cerebrovascular "Corresponding author.

How to cite this paper: Liu, L. and Zhou, Y.Y. (2015) Effect of Carotid Artery Stenting and Extracranial-Intracranial By-Pass on Cognitive Function: Preliminary Results. International Journal of Clinical Medicine, 6, 377-383.

http://dx.doi.org/10.4236/ijcm.2015.66049 
disease, and has been demonstrated to be associated with stroke [1], cognitive impairment [2] [3] and dementia [4]. It can reduce cerebral perfusion [5] [6], which further causes oxidative stress, mitochondrial dysfunction, and neuroinflammation [7] [8], and eventually leads to neuropathological changes and cognitive performance decline. To reduce the risk of stroke, carotid artery stenting (CAS) is performed and has shown to be effective in preventing from stroke in patients with severe carotid artery stenosis. For patients with intracranial stenosis, especially caused by intracranial atherosclerotic disease (ICAD), medical intervention is required to reduce the risk of ischemic stroke due to thromboembolic events, but it does not reduce the risk of ICAD progression, hypoperfusion and poor collateral circulation. Therefore extracranial-intracranial (EC-IC) bypass was developed to reduce the risk of ischemic stroke in patients with impaired cerebral hemodynamics due to occlusive cerebrovascular disease. Several studies have shown that restoration of blood flow after CAS or EC-IC by-pass may improve cognitive function [9]-[12]. Conversely some authors had concerns that revascularization may have a negative impact on cognition caused by procedural emboli, general anesthesia, or temporary flow interruption [13]. Further studies are needed to confirm the role of revascularization on cognition. The goal of this retrospective study was to evaluate cognitive performance in patients following CAS and EC-IC by-pass.

\section{Materials and Methods}

\subsection{Study Patients}

Consecutive patients who were referred to neuroendovascular unit for carotid artery stent placement or EC-IC by-pass from January 2010 to October 2011 participated in the study. The institutional review board approved the study. The degree of carotid and intracranial stenosis was initially evaluated by CT angiogram (CTA), and conventional cerebral angiography was followed in anticipation of CAS and EC-IC by-pass. Brain MR perfusion-weighted imaging was performed in all subjects. Inclusion criteria included recent (within 30 days of the date of procedure) CTA of the head and neck, evidence of an carotid stenosis of more than $70 \%$ confirmed with conventional angiography or severe intracranial major artery stenosis with distinct low perfusion of the relevant regions, age older than 45 years, and selected by the vascular disease specialist as a suitable candidate for stent placement or EC-IC by-pass. CAS was performed if the patients had severe symptomatic carotid stenosis $\geq 70 \%$ without severe diffused intracranial artery stenosis. The indications for EC-IC bypass surgery were haemodynamic compromise due to atherosclerotic occlusive cerebrovascular disease which was demonstrated by the MRI perfusion imaging. Controls were patients with carotid or/and intracranial severe stenosis who declined vascular surgery due to personal reasons or no surgery was possible due to medical reasons. Exclusion criteria included evidence of a previous large stroke or cerebral infarction, a history of previous subarachnoid or cerebral hemorrhage, intracranial arteritis and cerebral arteriopathy. All patients including controls received aspirin $100 \mathrm{mg}$ and clopidogrel $75 \mathrm{mg}$ daily after the procedure.

\subsection{Methods}

We collected demographic information and the vascular risk factors of each patient. Neuropsychologic testing was conducted by a trained research assistant. Global cognitive function was assessed using the Mini-Mental State Examination (MMSE) and Montreal cognitive assessment (MoCA), self-care abilities were assessed with Activities of Daily Living (ADLs), and patient's mood was assessed with the Hamilton Rating Scale for Depression (HMLD). Both MMSE and MoCA are screening tests for assessing the cognitive state of patients and are simple to use, sensitive, and valid. From the inclusion into clinical practice they have been proven as reliable and suitable for the initial assessment of mental status follow-up. The MMSE examines the temporal and spatial orientation, memory skills (immediate and delayed), attention, oral and written language, and constructional abilities in two dimensions. The MMSE has eleven tasks where each one scores a number of points, total score is 30 points, and the scale ranges from 0 to 30, so that there are levels of severe cognitive impairment (from 0 to 17 points); medium impairment (from 18 to 23 points); and without impairment (from 24 to 30 points). The MoCA investigates patient's skills in 5 domains: visuospatial/executive, naming, memory, attention, abstraction, and orientation. The total score is the sum of all items, with a maximum score of 30 (best performance). The ADL is a carer rated instrument consisting of 20 daily-living ability items. For each item, four dimensions on initiation, organization and effectiveness to carry out a specific task would be measured to give an overall rating. HMLD assesses depression through 21 questions. The higher the score, the more severe the depression as follows: 0 - 7 no depression; 8 - 13 mild depression; 14 - 18 moderate depression; 19 - 22 severe depression; $\geq 23$ very severe 
depression. All Assessments were performed before and 3 months after surgery.

\subsection{Statistical Analysis}

All measurement data are presented as mean \pm SD or median \pm quartile as appropriate. Categorical data are given as counts. Chi-square tests were performed to compare categorical variables such as gender, history and risk factors in cross tables. One-way ANOVA were performed to compare measurement baseline characteristics. We used a paired Student t test to compare neuropsychologic test scores at baseline and at three months after procedure in each group. Cognitive assessment score changes in a three-month follow-up were not normally distributed and compared among/between groups with Kruskal-Wallis Test. Differences were deemed statistically significance if $P<0.05$. We performed all statistical analyses with SPSS version 13.0 (SPSS, Chicago, IL).

\section{Results}

We totally enrolled 118 patients into the study. Of the 118 patients, 43 were performed CAS, 32 were performed EC-IC by-pass, and 43 conformed to CAS/EC-IC criteria but declined any surgery. No periprocedural adverse clinical events were noted. Demographics, clinical symptoms and vascular risk factors except for age showed no significant differences among the CAS, EC-IC by-pass and control groups. There were younger patients in the EC-IC by-pass group. There were no significant differences in baseline MMSE, MoCA, ADLs and HMLD scores among the groups (Table 1).

The MMSE and MoCA scores did not significantly change from baseline in the control patients at threemonth follow-up $(P=0.323$ and 0.685$)$. In CAS group, the total scores for the MMSE and MoCA increased significantly to 26.37 and 23.23 three months after stent placement comparing to the baseline average 25.16 and $21.37(P<0.001)$. As to EC-IC by-pass group, the average MMSE and MoCA scores also increased significantly to 26.59 and 24.13 from 25.13 and 21.88 three months after EC-IC by-pass surgery (Table 2). The changes on the MMSE and MoCA scores from baseline differed significantly among the three groups (Table 3). Both CAS and EC-IC by-pass groups had improvement on cognitive performance compared to the control group (Table 4). Comparison of the change scores for the MMSE and MoCA showed no significant differences between the CEA and EC-IC by-pass groups ( $P=0.264$ and 0.289 respectively).

Both the ADLs and HMLD significantly improved from baseline in all three groups after three months (Table 2), and EC-IC group presented a greater recovery in daily life abilities comparing to the other two groups (Table 3, Table 4). There were no significant differences in the changes of HMLD scores among three groups (Table 3).

\section{Discussion}

The principal aim of our investigation was to determine whether patients experienced improved cognition following CAS or EC-IC by-pass. In our study, patients before stent placement had, on average, total MMSE score of 25.51 and MoCA score of 22.01, with which MoCA was lower than the normal value. The scores for both MMSE and MoCA increased significantly after stent placement. The same cognitive improvement effect was also seen in EC-IC by-pass group, whereas there was no significant difference comparing to baseline after three months in the control group.

CAS is believed to prevent stroke as a less invasive alternative to carotid endarterectomy (CEA) in selected patients with symptomatic carotid stenosis [14] [15], but its influence on cognitive performance in patients remains controversial [16] [17]. The results in our study agreed with the results from previous reports which have shown improvements in cognitive function in patients treated with carotid stent placement [18]-[22]. In addition, the average MoCA score of the patients from all three groups was below the normal value. To data, there has not been a significant study of the incidence of cognitive dysfunction in patients with carotid artery stenosis. It is speculative that majority of patients with severe carotid and/or intracranial major artery stenosis experience cognitive impairment to some degree. The possible mechanisms of a cognitive impairment include silent embolization and hypoperfusion in brain. Carotid stenting may lead to an increment and restoration in brain perfusion, and eventually improve patients' cognitive function. The reports suggesting a potential cognitive deterioration from CAS were mostly in the earlier introduction of carotid stent before emboli-protection devices (EPDs) were developed [23] [24]. The post-CAS cognitive dysfunction was thought to be a consequence of microembolic ischemia or intraprocedural hypoperfusion. In all our CAS patients, a distal filter was deployed during the procedure, which reduced the risk of stent-related particle emboli and strokes. 
Table 1. Demographics, vascular risk factors and baseline cognitive function in the CAS, EC-IC by-pass and control groups.

\begin{tabular}{cccccc}
\hline & CAS $(\mathrm{n}=43)$ & EC-IC $(\mathrm{n}=32)$ & Control $(\mathrm{n}=43)$ & $\mathrm{F} / \chi^{2}$ & $P$ \\
\hline Age & $63.26 \pm 8.353$ & $56.44 \pm 9.098$ & $62.53 \pm 8.192$ & 6.803 & 0.002 \\
Gender (female/male) & $18 / 25$ & $12 / 20$ & $25 / 18$ & 3.754 & 0.153 \\
Education (yrs) & $8.63 \pm 3.684$ & $9.69 \pm 3.207$ & $9.23 \pm 3.265$ & 0.914 & 0.404 \\
Handedness (right/left) & $41 / 2$ & $32 / 1$ & $41 / 2$ & 0.134 & 0.935 \\
Hypertension & 34 & 21 & 32 & 1.792 & 0.421 \\
Cardiac disease & 16 & 11 & 14 & 0.208 & 0.901 \\
Diabetes & 12 & 14 & 14 & 2.109 & 0.348 \\
Stroke & 31 & 18 & 12 & 2.466 & 0.291 \\
Smoke & 33 & 18 & 13 & 1.691 & 0.429 \\
Drink & 29 & 11 & 3.527 & 0.171 \\
BMI & $25.57 \pm 2.736$ & $25.69 \pm 2.666$ & $25.02 \pm 2.445$ & 0.738 & 0.480 \\
MMSE & $25.16 \pm 3.866$ & $25.13 \pm 3.180$ & $26.14 \pm 2.455$ & 1.298 & 0.277 \\
MoCA & $21.37 \pm 5.790$ & $21.88 \pm 4.218$ & $22.74 \pm 3.317$ & 0.980 & 0.379 \\
HMLD & $5.60 \pm 3.704$ & $5.84 \pm 3.409$ & $6.77 \pm 3.184$ & 1.342 & 0.265 \\
ADLs & $27.23 \pm 10.585$ & $29.22 \pm 10.162$ & $27.98 \pm 9.728$ & 0.352 & 0.704 \\
\hline
\end{tabular}

BMI: body mass index; MMSE: mini-mental state examination; MoCA: Montreal cognitive assessment; ADLs: activities of daily living; HMLD: Hamilton rating scale for depression.

Table 2. Neuropsychologic test scores at baseline and follow-up.

\begin{tabular}{|c|c|c|c|c|c|}
\hline & & Baseline & 3 months & $\mathrm{t}$ & $P$ \\
\hline \multirow[t]{4}{*}{ Control } & MMSE & $26.14 \pm 2.455$ & $26.02 \pm 2.335$ & 1.000 & 0.323 \\
\hline & MoCA & $22.74 \pm 3.317$ & $22.84 \pm 3.177$ & -0.409 & 0.685 \\
\hline & ADLs & $27.98 \pm 9.728$ & $24.53 \pm 3.686$ & 2.375 & 0.022 \\
\hline & HMLD & $6.77 \pm 3.184$ & $5.07 \pm 2.658$ & 3.907 & $<0.001$ \\
\hline \multirow[t]{4}{*}{ CAS } & MMSE & $25.16 \pm 3.866$ & $26.37 \pm 3658$ & -5.061 & $<0.001$ \\
\hline & MoCA & $21.37 \pm 5.790$ & $23.23 \pm 5.227$ & -4.699 & $<0.001$ \\
\hline & ADLs & $27.23 \pm 10.585$ & $23.37 \pm 7.499$ & 2.267 & 0.029 \\
\hline & HMLD & $5.60 \pm 3.704$ & $3.60 \pm 3.685$ & 3.631 & 0.001 \\
\hline \multirow[t]{4}{*}{ EC-IC } & MMSE & $25.13 \pm 3.180$ & $26.59 \pm 2.698$ & -5.239 & $<0.001$ \\
\hline & MoCA & $21.88 \pm 4.218$ & $24.13 \pm 3.230$ & -4.962 & $<0.001$ \\
\hline & ADLs & $29.22 \pm 10.162$ & $24.09 \pm 6.249$ & 4.082 & $<0.001$ \\
\hline & HMLD & $5.84 \pm 3.409$ & $3.78 \pm 2.779$ & 3.524 & 0.001 \\
\hline
\end{tabular}

BMI: body mass index; MMSE: mini-mental state examination; MoCA: Montreal cognitive assessment; ADLs: activities of daily living; HMLD: Hamilton rating scale for depression.

Table 3. Changes of neuropsychologic assessment scores at a 3-month follow up in the CAS, EC-IC by-pass and control groups.

\begin{tabular}{ccccc}
\hline & CAS $(\mathrm{n}=43)$ & EC-IC $(\mathrm{n}=32)$ & Control $(\mathrm{n}=43)$ & $P$ \\
\hline MMSE & $1.2093 \pm 1.5669$ & $1.4688 \pm 1.5859$ & $-0.1163 \pm 0.7625$ & $<0.001$ \\
MoCA & $1.8605 \pm 2.5965$ & $2.2500 \pm 2.5653$ & $0.0000 \pm 1.3973$ & $<0.001$ \\
ADLs & $-3.8605 \pm 11.1667$ & $-5.1250 \pm 7.1018$ & $-3.4419 \pm 9.5026$ & 0.011 \\
HMLD & $-2.0000 \pm 3.2450$ & $-2.0625 \pm 3.3112$ & $-1.6977 \pm 2.8497$ & 0.597 \\
\hline
\end{tabular}

BMI: body mass index; MMSE: mini-mental state examination; MoCA: Montreal cognitive assessment; ADLs: activities of daily living; HMLD: Hamilton rating scale for depression. 
Table 4. Comparison of neuropsychologicassessment changes of both CAS and EC-IC by-pass patients with the controls.

\begin{tabular}{ccc}
\hline & $P$ & $P$ \\
CAS $(\mathrm{n}=43)$ & EC-IC $(\mathrm{n}=32)$ \\
MMSE & $<0.001$ & $<0.001$ \\
MoCA & $<0.001$ & $<0.001$ \\
ADLs & 0.084 & 0.002 \\
HMLD & 0.378 & 0.394
\end{tabular}

BMI: body mass index; MMSE: mini-mental state examination; MoCA: Montreal cognitive assessment; ADLs: activities of daily living; HMLD: Hamilton rating scale for depression.

EC-IC bypass surgery has been used to increase the cerebral blood flow and reduce the risk of future strokes in patients with ischemic cerebrovascular disease [25] [26]. With the cooperative study on EC-IC bypass in 1985 failing to show a benefit from the bypass procedure over medical management for anterior circulation occlusive disease [27], the bypass procedure was almost abandoned by the mainstream neurosurgeons. Despite the disappointing findings of this study, a subpopulation of patients with ischemic vascular disease and poor hemodynamic reserve may benefit from EC-IC bypass. Given the above reason, a trail was carried out to re-evaluate the EC-IC bypass procedure. To our knowledge, there were few studies to explore the cognition changes after by-pass surgery. Our results showed that cognitive function improved in the patients with intracranial vascular stenosis and hemodynamic compromise three months after EC-IC by-pass. Besides, EC-IC bypass and CAS did not have the different outcome on cognitive performance. In our experience, the cognition improvement somehow was particularly apparent in the patients' language. But we didn't include verbal fluency tests in the patient's neuropsychologic assessments.

We compared both ADLs and HMLD in all patients in a three-month follow-up. There was a significant improvement in instrumental daily living abilities and Hamilton depression scores in all the three groups, meanwhile EC-IC by-pass showed a superior effect on daily life abilities. Except for distinct advantage of EC-IC by-pass over patients' living ability, all three treatments including CAS, EC-IC by-pass and medications were equally effective in improvement of patients' living function and depression. Activities of daily living and neuropsychiatric amelioration may result from the cognitive improvement, the medication, or the spontaneous alleviation during the course of the cerebral ischemia.

There were few studies of the effects of both EC-IC by-pass and CAS on cognitive performance in patients with carotid and intracranial stenosis. We defined the patients who conformed to CAS/EC-IC by-pass criteria but declined any surgery as a control group, which greatly avoided the influence of some confounding factors on the results. But we only assessed neuropsychological function with the global cognitive tests, and some specific cognitive domains (i.e., attention and language, executive function) were not being evaluated with the pertinent neuropsychological examinations. Additionally, our study sample was small. Given the limited number of patients included in the analysis, our results should be considered as preliminary. A future study with larger patient populations and more specific cognitive assessments would be conducted to confirm these preliminary findings.

\section{Conclusion}

The present study demonstrated that both CAS and EC-IC by-pass resulted in increased cognitive performance three months after procedures. Considering the limitation on sample size, further work is needed to confirm our current findings. With the widespread applying revascularization and increasing population of vascular cognitive impairment, it is important for randomized, controlled trials evaluating the benefits and risks of the procedures to include cognitive function assessments. Furthermore, understanding the effect of carotid and cerebral revascularization on cognition might help to reduce the incidence of vascular dementia.

\section{References}

[1] Barnett, H.J., Taylor, D.W., Eliasziw, M., et al. (1998) Benefit of Carotid Endarterectomy in Patients with Symptomatic Moderate or Severe Stenosis. North American Symptomatic Carotid Endarterectomy Trial Collaborators. The New England Journal of Medicine, 339, 1415-1425. http://dx.doi.org/10.1056/NEJM199811123392002

[2] Mathiesen, E.B., Waterloo, K., Joakimsen, O., et al. (2004) Reduced Neuropsychological Test Performance in Asympto- 
matic Carotid Stenosis: The Tromso Study. Neurology, 62, 695-701.

http://dx.doi.org/10.1212/01.WNL.0000113759.80877.1F

[3] Romero, J.R., Beiser, A., Seshadri, S., et al. (2009) Carotid Artery Atherosclerosis, MRI Indices of Brain Ischemia, Aging, and Cognitive Impairment: The Framingham Study. Stroke, 40, 1590-1596.

http://dx.doi.org/10.1161/STROKEAHA.108.535245

[4] De la Torre, J.C. (2004) Is Alzheimer's Disease a Neurodegenerative or a Vascular Disorder? Data, Dogma, and Dialectics. Lancet Neurology, 3, 184-190. http://dx.doi.org/10.1016/S1474-4422(04)00683-0

[5] Norris, E.J. (2010) Anesthesia for Vascular Surgery. In: Miller, R.D., Ed., Miller's Anesthesia, Churchill Livingstone Elsevier, Philadelphia. http://dx.doi.org/10.1016/B978-0-443-06959-8.00062-5

[6] Derdeyn, C.P. (2007) Mechanisms of Ischemic Stroke Secondary to Large Artery Atherosclerotic Disease. Neuroimaging Clinics of North America, 17, 303-311. http://dx.doi.org/10.1016/j.nic.2007.03.001

[7] Zlokovic, B.V. (2005) Neurovascular Mechanisms of Alzheimer’s Neurodegeneration. Trends in Neurosciences, 28, 202-208. http://dx.doi.org/10.1016/j.tins.2005.02.001

[8] Nishio, K., Ihara, M., Yamasaki, N., et al. (2010) A Mouse Model Characterizing Features of Vascular Dementia with Hippocampal Atrophy. Stroke, 41, 1278-1284. http://dx.doi.org/10.1161/STROKEAHA.110.581686

[9] Lin, M.S., Chiu, M.J., Wu, Y.W., et al. (2011) Neurocognitive Improvement after Carotid Artery Stenting in Patients with Chronic Internal Carotid Artery Occlusion and Cerebral Ischemia. Stroke, 42, 2850-2854. http://dx.doi.org/10.1161/STROKEAHA.111.613133

[10] Lal, B.K., Younes, M., Cruz, G., et al. (2011) Cognitive Changes after Surgery vs Stenting for Carotid Artery Stenosis. Journal of Vascular Surgery, 54, 691-698. http://dx.doi.org/10.1016/j.jvs.2011.03.253

[11] Grunwald, I.Q., Papanagiotou, P., Reith, W., Backens, M., Supprian, T., Politi, M., et al. (2010) Influence of Carotid Artery Stenting on Cognitive Function. Neuroradiology, 52, 61-66. http://dx.doi.org/10.1007/s00234-009-0618-4

[12] Dong, Y.H., Teoh, H.L., Chan, B.P.L., Ning, C., Yeo, T.T., Sinha, A.K., et al. (2012) Changes in Cerebral Hemodynamic and Cognitive Parameters after External Carotid-Internal Carotid Bypass Surgery in Patients with Severe Steno-Occlusive Disease: A Pilot Study. Journal of the Neurological Sciences, 322, 112-116. http://dx.doi.org/10.1016/j.jns.2012.07.034

[13] Bendszus, M. and Stoll, G. (2006) Silent Cerebral Ischaemia: Hidden Fingerprints of Invasive Medical Procedures. The Lancet Neurology, 5, 364-572. http://dx.doi.org/10.1016/S1474-4422(06)70412-4

[14] Brott, T.G., Hobson II, R.W., Howard, G., Roubin, G.S., Clark, W.M., Brooks, W., et al. (2010) Stenting versus Endarterectomy for Treatment of Carotid-Artery Stenosis. New England Journal of Medicine, 363, 11-23. http://dx.doi.org/10.1056/NEJMoa0912321

[15] Hassoun, H.T., Malas, M.B. and Freischlag, J.A. (2010) Secondary Stroke Prevention in the Era of Carotid Stenting: Update on Recent Trials. Archives of Surgery, 145, 928-935. http://dx.doi.org/10.1001/archsurg.2010.204

[16] Gaudet, J.G., Meyers, P.M. and McKinsey, J.F. (2009) Incidence of Moderate to Severe Cognitive Dysfunction in Patients Treated with Carotid Artery Stenting. Neurosurgery, 65, 325-330. http://dx.doi.org/10.1227/01.NEU.0000349920.69637.78

[17] De Rango, P., Caso, V., Leys, D., Paciaroni, M., Lenti, M. and Cao, P. (2008) The Role of Carotid Artery Stenting and Carotid Endarterectomy in Cognitive Performance: A Systematic Review. Stroke, 39, 3116-3127. http://dx.doi.org/10.1161/STROKEAHA.108.518357

[18] Turk, A.S., Chaudry, I., Haughton, V.M., Hermann, B.P., Rowley, H.A., Pulfer, K., et al. (2008) Effect of Carotid Artery Stenting on Cognitive Function in Patients with Carotid Artery Stenosis: Preliminary Results. American Journal of Neuroradiology, 29, 265-268. http://dx.doi.org/10.3174/ajnr.A0828

[19] Mendiz, O.A., Sposato, L.A., Fabbro, N., Lev, G.A., Calle, A., Valdivieso, L.R., et al. (2012) Improvement in Executive Function after Unilateral Carotid Artery Stenting for Severe Asymptomatic Stenosis. Journal of Neurosurgery, 116, 179-184. http://dx.doi.org/10.3171/2011.9.JNS11532

[20] Grunwald, I.Q., Supprian, T., Politi, M., Struffert, T., Falkai, P., Krick, C., et al. (2006) Cognitive Changes after Carotid Artery Stenting. Neuroradiology, 48, 319-323. http://dx.doi.org/10.1007/s00234-006-0064-5

[21] Xu, G.L., Liu, X.F., Meyer, J.S., Yin, Q. and Zhang, R. (2007) Cognitive Performance after Carotid Angioplasty and Stenting with Brain Protection Devices. Neurological Research, 29, 251-255. http://dx.doi.org/10.1179/016164107X159216

[22] Kostopanagiotou, G., Markantonis, S.L., Polydorou, M., Pandazi, A. and Kottis, G. (2005) Recovery and Cognitive Function after Fentanyl or Remifentanil Administration for Carotid Endarterectomy. Journal of Clinical Anesthesia, 17, 16-20. http://dx.doi.org/10.1016/j.jclinane.2004.03.008

[23] Kastrup, A., Gröschel, K., Krapf, H., Brehm, B.R., Dichgans, J. and Schulz, J.B. (2003) Early Outcome of Carotid An- 
gioplasty and Stenting with and without Cerebral Protection Devices: A Systematic Review of the Literature. Stroke, 34, 813-819. http://dx.doi.org/10.1161/01.STR.0000058160.53040.5F

[24] Eskandari, M.K. (2005) Cerebral Embolic Protection. Seminars in Vascular Surgery, 18, 95-100. http://dx.doi.org/10.1053/j.semvascsurg.2005.04.006

[25] Yoshimoto, Y. and Kwak, S. (1995) Superficial Temporal Artery-Middle Cerebral Artery Anastomosis for Acute Cerebral Ischemia: The Effect of Small Augmentation of Blood Flow. Acta Neurochirurgica, 137, 128-137. http://dx.doi.org/10.1007/BF02187184

[26] Pikus, H.J. and Heros, R.C. (1999) Stroke: Indications for Emergent Surgical Intervention. Clinical Neurosurgery, 45, 113-127.

[27] EC-IC Bypass Study Group (1985) Failure of Extracranial-Intracranial Arterial Bypass to Reduce the Risk of Ischemic Stroke: Results of an International Randomized Trial. New England Journal of Medicine, 313, 1191-1200. http://dx.doi.org/10.1056/NEJM198511073131904 